\title{
DISTRIBUCIÓN ESPACIAL DE Fe Li, Pb, Mn, V, Y Zn EN SUELOS URBANOS DE MORELIA, MICHOACÁN, MÉXICO
}

\author{
Carmen DELGADO ${ }^{*}$, Isabel ISRADE ALCÁNTARA ${ }^{2}$, Francisco BAUTISTA ${ }^{3}$, Avto GOGICHAISHVILI ${ }^{1}$, \\ Ciro MÁRQUEZ ${ }^{4}$, Rubén CEJUDO ${ }^{1}$, Juan MORALES ${ }^{1}$ e Isamar GONZÁLEZ ${ }^{5}$
}

${ }^{1}$ Laboratorio Universitario de Geofísica Ambiental, Instituto de Geofísica, Unidad Michoacán, Universidad Nacional Autónoma de México. Antigua Carretera a Pátzcuaro No. 8701, Colonia Ex-Hacienda de San José de la Huerta. Morelia, Michoacán, México, C. P. 58190

${ }^{2}$ Instituto de Investigaciones en Ciencias de la Tierra, Universidad Michoacana de San Nicolás de Hidalgo. Ciudad Universitaria, Edificio U-III. Francisco J. Mújica, Colonia Felícitas del Río, Morelia, Michoacán, México, C. P. 58030

${ }^{3}$ Laboratorio Universitario de Geofísica Ambiental, Centro de Investigaciones en Geografía Ambiental, Universidad Nacional Autónoma de México. Antigua Carretera a Pátzcuaro No. 8701, Colonia Ex-Hacienda de San José de la Huerta, Morelia, Michoacán, México, C. P. 58190

${ }^{4}$ Facultad de Química, Universidad Nacional Autónoma de México. Avenida Universidad 3000, Circuito exterior, Colonia Universidad, Ciudad de México, México, C. P. 04510

${ }^{5}$ Instituto Tecnológico Superior de Tacámbaro. Avenida Tecnológico 201, Zona El Gigante, Tacámbaro, Michoacán, México, C. P. 61650

*Autor para correspondencia: meladc56@yahoo.com.mx

(Recibido enero 2017; aceptado octubre 2017)

Palabras clave: contaminación, metales pesados, Normas Oficiales Mexicanas, geoestadística

\section{RESUMEN}

Morelia Michoacán, México es la ciudad más poblada del estado Michoacán, con alto tráfico vehicular y poca industrialización. En Morelia se ha reportado la presencia de elementos potencialmente tóxicos (EPT) en los suelos urbanos entre los cuales el Fe, $\mathrm{Mn}, \mathrm{Pb}$ y V rebasaron los límites establecidos por las normas ambientales mexicanas y estadounidenses. Para la toma de decisiones se requiere la localización de los sitios de mayor contaminación mediante la elaboración de mapas con la distribución espacial de los EPT. El objetivo de este trabajo fue analizar la distribución espacial de Fe, $\mathrm{Li}$, $\mathrm{Mn}, \mathrm{Pb}, \mathrm{V}$ y $\mathrm{Zn}$ en los suelos urbanos de Morelia para identificar las zonas de mayor concentración. Se colectaron cien muestras de suelo superficial. La concentración de los EPT fue medida con espectrometría de plasma acoplado inductivamente de tipo óptico. Se hizo un análisis geoestadístico con kriging ordinario $(\mathrm{KO})$ para conocer la distribución de los EPT y con kriging indicador (KI) para identificar las zonas con mayor probabilidad de contaminación. Los variogramas del Fe, Mn, Li y Zn con KO mostraron valores de $\mathrm{R}^{2} \geq 0.8$. Las zonas con mayor probabilidad de rebasar las normas ambientales son: el centro por $\mathrm{Fe}$ y $\mathrm{Pb}$, el este por $\mathrm{Fe}$ y $\mathrm{V}$, el suroeste por Mn y el oeste por Fe. En el centro y este de la ciudad existe la mayor contaminación.

Key words: contamination, heavy metals, Official Mexican standards, geoestatistics 


\begin{abstract}
Morelia, Michoacán, Mexico is the most populated city in the state of Michoacán, with high vehicular traffic and little industrialization. In Morelia, the presence of potentially toxic elements (PTE) has been reported in urban soils, among which $\mathrm{Fe}, \mathrm{Mn}, \mathrm{Pb}$ and $\mathrm{V}$ exceeded the limits established by the Mexican and US environmental standards. For the decision making it is necessary the location of the sites of greater contamination by the elaboration of maps with the spatial distribution of the PTE. The principal aim of this work was to analyze the spatial distribution of $\mathrm{Fe}, \mathrm{Li}, \mathrm{Mn}, \mathrm{Pb}, \mathrm{V}$ and $\mathrm{Zn}$ in the urban soils of Morelia to identify the areas with the highest concentration. In total, one hundred of top soil samples were collected. The analysis of the PTE was performed by inductively coupled optical plasma spectrometry. A geostatistical analysis was performed with ordinary kriging $(\mathrm{OK})$ to know the distribution of PTE and with indicator kriging to identify the areas with the highest probability of contamination. The models of the variograms of $\mathrm{Fe}, \mathrm{Mn}, \mathrm{Li}$ and $\mathrm{Zn}$ with $\mathrm{OK}$ yielded values of $\mathrm{R}^{2} \geq 0.8$. The areas most likely to exceed environmental norms are: the dowtown for $\mathrm{Fe}$ and $\mathrm{Pb}$, the east by Fe and V, the southwest by Mn and the west by Fe. In the downtown and east of the city there is the greatest contamination.
\end{abstract}

\section{INTRODUCCIÓN}

La contaminación del suelo urbano con elementos potencialmente tóxicos (EPT), que se dispersan por diferentes vías, proviene de las actividades humanas como la industria ( $\mathrm{Su}$ y Wong 2003, Madrid et al. 2008, Testiati et al. 2013), el tráfico vehicular (Aguilar et al. 2012, Sánchez-Duque et al. 2015) y la construcción (Morton et al. 2001, Chen et al. 2005).

El ingreso de los EPT a las cadenas alimentarias depende de la fuente de emisión, el $\mathrm{pH}$, la solubilidad y tamaño de la partícula que contiene a los EPT que los hace biodisponibles y con riesgo para la salud humana (Rawlins et al. 2005, Jordao et al. 2006, Laing et al. 2014).

El $\mathrm{Pb}$ es nefrotóxico (Granero y Domingo 2002, Sabath y Robles-Osorio 2012). El Mn daña el sistema nervioso, el $\mathrm{V}$ el cardiorrespiratorio, nervioso y digestivo, el Fe y el Li el ocular y respiratorio y el $\mathrm{Zn}$ el digestivo y cardiovascular (ATDSR 2010, Chen et al. 2015, Lenntech 2016). En México existe legislación ambiental para prever efectos en la salud.

La legislación ambiental mexicana mediante la norma oficial mexicana NOM-147-SEMARNAT/ SSA1-2004 (SEMARNAT 2007) establece los límites máximos permitidos (LMP) en suelo de uso residencial para el $\mathrm{Pb}(400 \mathrm{mg} / \mathrm{kg})$ y V $(78 \mathrm{mg} / \mathrm{kg})$. La legislación estadounidense los establece para el $\mathrm{Fe}(23000 \mathrm{mg} / \mathrm{kg}), \mathrm{Li}(1600 \mathrm{mg} / \mathrm{kg}), \mathrm{Mn}(1800 \mathrm{mg} /$ $\mathrm{kg})$ y Zn (23 $000 \mathrm{mg} / \mathrm{kg})$ (USEPA 2004).
En estudios previos de contaminación de suelos por EPT en Morelia, Michoacán, se concluyó que provienen del tráfico vehicular y vialidades primarias (Aguilar et al. 2013); el Pb y V rebasaron los LMP por la NOM-147-SEMARNAT/SSA1-2004 (SEMARNAT 2007) y el Fe y Mn los establecidos por la USEPA (2004). No se encontraron diferencias significativas por tipos de uso de suelo o vialidad (Delgado et al. 2015).

Se requiere la elaboración de mapas precisos de las zonas contaminadas para ser atendidas por las autoridades mediante intervenciones efectivas de saneamiento ambiental.

El diseño de mapas precisos con validez matemática, que muestren la distribución espacial de los contaminantes, se puede realizar mediante métodos geoestadísticos como el kriging ordinario que tiene mínimo error en la estimación. La limitante es que sólo proporciona una visión general de la distribución espacial sin delimitar los niveles de probabilidad de rebasar un LMP.

Con el método kriging indicador se pueden señalar en el mapa los sitios precisos con los diferentes niveles de probabilidad de rebasar los LMP. Este método consiste de una transformación binaria de los datos (0-1) para que el estimador sea menos sensible a los datos extremos (Webster y Oliver 1990, Goovaerts 1999).

El objetivo de este trabajo fue analizar la distribución espacial de $\mathrm{Fe}$, Li, Mn, $\mathrm{Pb}, \mathrm{V}$ y Zn en los suelos urbanos de la ciudad de Morelia y la zona conurbada para identificar las zonas de mayor concentración 
mediante dos técnicas de interpolación: kriging ordinario y kriging indicador.

\section{MATERIALES Y MÉTODOS}

La ciudad de Morelia se ubica en el centro-norte del estado de Michoacán, a 1920 msnm. El clima es templado subhúmedo con una temperatura media anual de $17.5^{\circ} \mathrm{C}$ y una precipitación de $773 \mathrm{~mm}$ anuales. Los vientos dominantes proceden del suroeste y noroeste. Presenta 648 ha de reservas ecológicas urbanas y 134 ha de áreas verdes Los grupos de suelos son: Vertisoles, Luvisoles, Acrisoles, Leptosoles y Andosoles (PDUCPM 2010).

El basamento de la ciudad está conformado por rocas ignimbritas; en el este y oeste existe una secuencia de depósitos lacustres, fluviolacustres y epiclásticos del Mioceno superior y Plioceno superior (Garduño-Monroy et al. 1999).

El municipio de Morelia tiene las siguientes actividades económicas: comercio, turismo y servicios $(63.7 \%)$, industria de la construcción y manufacturera $(25.9 \%)$, agricultura $(10.4 \%)$ (INECCSEMARNAT 2010).
El muestreo se realizó en la ciudad de Morelia y su área conurbada colindante con el municipio de Tarímbaro, con 672069 habitantes, donde circulan 332381 automóviles (INEGI 2010, 2014) cuya verificación de los gases generados no es obligatoria (INECC-SEMARNAT 2010).

\section{Muestreo}

El muestreo fue sistemático de 100 puntos, se realizó en febrero en época de secas (Fig. 1). Se colectó una muestra superficial de suelo bajo de árboles maduros. Se introdujo un cilindro de policloruro de vinilo de $100 \mathrm{~cm}^{3}$. Se retiró la hojarasca, piedras y gravas y el suelo se depositó en bolsas de plástico.

Las muestras se etiquetaron con las referencias geográficas registradas con un sistema geoposicionador (GPS) (Garmin GPS Map 76) calibrado bajo proyección Universal Transversal Mercator (UTM), elipsoide y datum horizontal Sistema Geodético Mundial (WGS84).

\section{Análisis químicos}

Las muestras de suelo se secaron a la sombra y temperatura ambiente; se tamizaron con malla de $2 \mathrm{~mm}$ y la porción fina se pesó, dividió en cuatro y
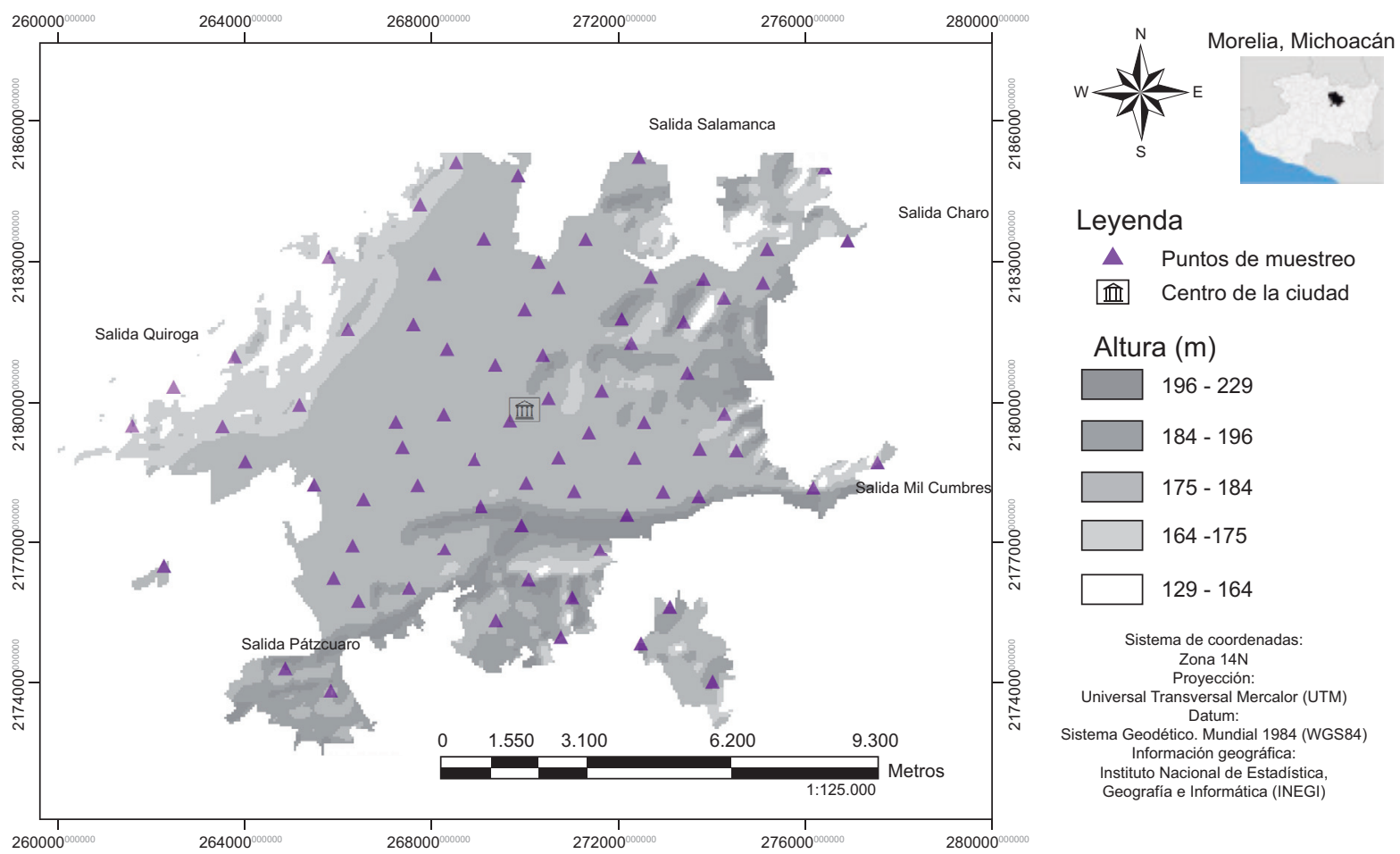

Centro de la ciudad

Altura (m)

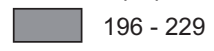

$184-196$

$\square 175-184$

$\square \quad 164-175$

$\square 129-164$

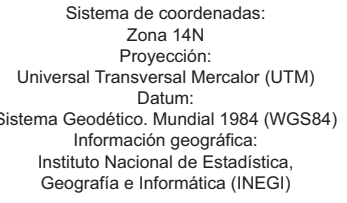

Fig. 1. Sitio de estudio, Morelia, Michoacán, México 
una porción se molió y tamizó con malla de $125 \mu \mathrm{m}$ (tamices de acero inoxidable W.S Tyler).

La digestión de las muestras se realizó en un horno de microondas (modelo MarsXpress ${ }^{\mathrm{TM}}$ ) de acuerdo con el método EPA 3051A, colocando 0.1 $\mathrm{g}$ de muestra de suelo con $\mathrm{HNO}_{3}$ y $\mathrm{H}_{2} \mathrm{O}_{2}(10: 1 \mathrm{~mL})$ en matraces con aforo de $100 \mathrm{~mL}$; el testigo fue sólo con reactivos.

La determinación de la concentración de los metales pesados fue mediante espectrometría de emisión con plasma acoplado inductivamente de tipo óptico (ICP-OES). Las concentraciones se reportaron en $\mathrm{mg} / \mathrm{kg}$.

Para asegurar la medición correcta de las muestras se realizó una curva de calibración con $0.0,0.1,1.0$ y $10 \mathrm{mg} / \mathrm{L}$ de $\mathrm{Pb}, \mathrm{Zn}, \mathrm{Fe}, \mathrm{Mn}, \mathrm{Li}, \mathrm{y} \mathrm{V}$ (r = 0.999). Al inicio y después de cada 40 mediciones se analizaron las soluciones estándar de 0.0 y $1.0 \mathrm{mg} / \mathrm{L}$.

\section{Análisis espacial}

Los mapas de la distribución espacial de los EPT se diseñaron a partir de un análisis geoestadístico utilizando el programa Geostatistics for the environmental sciences $\left(\mathrm{GS}^{+}\right)$(Robertson 2008) que consistió de las siguientes etapas:

1) Análisis exploratorio de datos para corroborar una distribución normal; en caso de no existir se hace algún tipo de transformación de los datos para mejorarla.

2) Autocorrelación espacial. Se ajusta el semivariograma a los datos medidos. Los criterios para la elección del modelo son: el coeficiente de determinación $\left(\mathrm{R}^{2}\right)$, el porcentaje de la varianza estructural y la distancia a la cual se alcanza la varianza máxima (D). El semivariograma se calcula de la manera siguiente:

$\gamma(h)=\frac{1}{2 N(h)} \sum_{i=1}^{N(h)}\left[Z\left(x_{i}\right)-Z\left(x_{i}+h\right)\right]^{2}$

En donde:

$\gamma(h)$ significa el valor del semivariograma experimental para todos los pares de datos del EPT a una distancia $h, Z\left(x_{i}\right)$ es la concentración del EPT en un sitio georreferenciado $x_{i}, Z\left(x_{i}+h\right)$ es la concentración del EPT en otro sitio separado de $x_{i}$ a una distancia $h, N(h)$ es el número de pares de sitios medidos y separados por una distancia $h$.

3) Estimación de los valores del EPT. Se hizo mediante kriging ordinario, utilizando los promedios ponderados a partir de la siguiente ecuación (Isaaks y Srivastava 1989, Webster y Oliver 1990, Hernández-Stefanoni y Ponce-Hernández 2006):
$Z\left(x_{0}\right)=\sum_{i=1}^{n} \lambda_{i} Z\left(x_{i}\right)$

En donde:

$\lambda_{i}$ es la suma de los pesos óptimos seleccionados, que debe ser igual a 1, a partir de un conjunto de $\mathrm{n}+1$ ecuaciones lineales simultáneas (Webster y Oliver 1990), $Z\left(x_{i}\right)$ son los valores medidos del EPT en un sitio y $Z\left(x_{0}\right)$ es la estimación no sesgada del EPT.

Los mapas del $\mathrm{Fe}, \mathrm{Mn}, \mathrm{Pb}$ y $\mathrm{V}$ también se realizaron con el método de interpolación kriging indicador para ubicar las zonas donde se rebasaron los LMP de acuerdo con la NOM 147-SEMARNAT/SSA1-2004 (SEMARNAT 2007) y USEPA (2004). El método acepta la no normalidad de los datos. El valor de 1 indica la máxima probabilidad de que la concentración del EPT sea igual o mayor que el LMP, los valores menores de 1 pero mayores de 0 indican algún grado de probabilidad de rebasarlo. Se calcula de la siguiente manera (Goovaerts 1999):

$I\left(x_{i} ; z_{k}\right)=\left\{\begin{array}{l}1, \text { cuando } Z\left(x_{i}\right) \leq Z_{k} \\ 0, \text { cuando } Z\left(x_{i}\right) \geq Z_{k}\end{array} \quad \mathrm{k}=1, \ldots, \mathrm{K}\right.$

En donde:

$I\left(x_{i} ; z_{k}\right)$ es la variable indicadora, $Z\left(x_{i}\right)$ es la variable medida y $Z_{k}$ es el umbral deseado.

El semivariograma indicador experimental,

$\lambda_{I}^{*}(h)=\frac{1}{2 N(h)} \sum_{i=1}^{N(h)}\left[I\left(x_{i} ; z_{k}\right)-I\left(x_{i}+h ; z_{k}\right)\right]^{2}$

En donde:

$\mathrm{N}(h)$ es el número de pares indicadores que transforman a $I\left(x_{i} ; z_{k}\right)$ e $I\left(x_{i}+h ; z_{k}\right)$ separados por una distancia $h$. La función de distribución acumulativa condicional en el sitio $x_{0}$ es:

$F\left(\frac{x_{0} ; z_{k}}{N}\right)=I *\left(x_{0} ; z_{k}\right)=\sum_{i=1}^{N} \lambda_{i} I\left(x_{0} ; z_{k}\right)$

En donde:

$I^{*}\left(x_{0} ; z_{k}\right)$ es el nivel del indicador estimado en el sitio no medido y $\lambda_{i}$ es el peso asignado al valor del indicador conocido $I\left(x_{i} ; z_{k}\right)$.

Los umbrales fueron los siguientes: $\mathrm{Pb}(400 \mathrm{mg} / \mathrm{kg})$, $\mathrm{V}(78 \mathrm{mg} / \mathrm{kg}), \mathrm{Mn}(1800 \mathrm{mg} / \mathrm{kg}) ; \mathrm{Fe}(23000 \mathrm{mg} / \mathrm{kg})$.

Finalmente, se realizó el mapa de integración donde se identificaron las zonas con mayor número de EPT con el nivel más alto $(0.8-1)$ de probabilidad de rebasar los LMP por las normas para que sean atendidas por las autoridades con mayor prioridad. Se usó el programa ArcGis 9 (ESRI 2004). 
CUADRO I. PARÁMETROS GEOESTADÍSTICOS (KRIGING ORDINARIO) DE LOS ELEMENTOS POTENCIALMENTE TÓXICOS EN LOS SUELOS URBANOS DE MORELIA, MICHOACÁN, MÉXICO

\begin{tabular}{lrcccr}
\hline EPT $(\mathrm{mg} / \mathrm{kg})$ & $\mathrm{n}$ & Semivariograma & $\mathrm{R}^{2}$ & Varianza estructural $(\%)$ & $\mathrm{D}(\mathrm{m})$ \\
\hline $\mathrm{Fe}$ & 100 & Exponencial & 0.9 & 61 & 12474 \\
$\mathrm{Zn}$ & 99 & Gaussiano & 0.9 & 65 & 8192 \\
$\mathrm{Mn}$ & 97 & Esférico & 0.8 & 70 & 3600 \\
$\mathrm{Li}$ & 99 & Exponencial & 0.8 & 61 & 12341 \\
$\mathrm{~V}$ & 80 & Esférico & 0.05 & 94 & 710 \\
$\mathrm{~Pb}$ & 74 & Gaussiano & 0.01 & 79 & 503 \\
\hline
\end{tabular}

$\mathrm{EPT}=$ elementos potencialmente tóxicos, $\mathrm{D}=$ distancia

\section{RESULTADOS Y DISCUSIÓN}

\section{La validez de las interpolaciones}

$\mathrm{El} \mathrm{Pb}$ y V tuvieron variogramas con valores bajos de $\mathrm{R}^{2} \mathrm{y}$ de distancia (D) (Cuadro I), lo cual indica que los modelos son tipo "efecto pepita" ya que la máxima varianza se alcanza a distancia muy corta y significa baja dependencia espacial y alta variabilidad debida al azar (Sommer et al. 2000, HernándezStefanoni y Ponce-Hernández 2006, Delgado et al. 2010). Por ello se descartaron para hacer mapas.

El Fe, Li, Mn y Zn tuvieron semivariogramas con valores de $\mathrm{R}^{2}$ mayores de 0.8 , lo que permitió elaborar mapas precisos de distribución espacial. La varianza estructural fue del 61 al $70 \%$, la distancia (D) de 3600 a $12474 \mathrm{~m}$, lo que indica que son los límites hasta donde los modelos explican la distribución espacial de los EPT (Cuadro I, Fig. 2).

\section{Mapas de las concentraciones de los EPT}

De acuerdo con los mapas de distribución de Fe, en Morelia se pudo observar que las concentraciones obtenidas (23000 hasta $41000 \mathrm{mg} / \mathrm{kg}$ ) rebasaron los LMP de la USEPA (2004) en el centro y el este de la ciudad con dirección al sur, hacia la salida a Mil cumbres (Fig. 3).

Las concentraciones de Fe deben estar por debajo de los LMP porque el Fe irrita las mucosas. Los óxidos de hierro causan neumoconiosis benigna, el hierro pentahidratado compite con organismos vivos por el oxígeno (Lenntech 2016). Lu et al. (2007) reportan valores máximos de $43000 \mathrm{mg} / \mathrm{kg}$ en suelos urbanos de Guangzhou, China, dos veces más altos que en Morelia.

El origen del Fe en los suelos de la zona centro de Morelia podría ser antrópico considerando el alto grado de densidad poblacional y el flujo vehicular. En la región este se encuentra la zona industrial más grande de la ciudad y alto flujo vehicular y en el sureste la densidad poblacional es media y con alto flujo vehicular.

En Guangzhou, China se reportan los mayores valores de $\mathrm{Fe}$ en los parques urbanos (45000 mg/kg) y con alto flujo vehicular (Lu et al. 2007). En Morelia, Delgado et al. (2015) encontraron valores de hasta $57000 \mathrm{mg} / \mathrm{kg}$ en el uso de suelo tipo área verde (AV).

El Zn presenta las concentraciones mayores en el centro de la ciudad (200 a $250 \mathrm{mg} / \mathrm{kg}$ ) y disminuye en las direcciones noroeste y sureste (Fig. 4). Es probable que su origen sea principalmente antrópico ya que se distribuye en zonas de alta y media densidad poblacional y con alto flujo vehicular, sin embargo, no rebasa los LMP de la USEPA (2004).

El Mn presenta las concentraciones mayores $(3640 \mathrm{mg} / \mathrm{kg})$ al suroeste de la ciudad en un área natural protegida pero que rodea a una industria papelera y $2202 \mathrm{mg} / \mathrm{kg}$ en un uso de suelo de baja densidad poblacional. También en el centro y centro-este las concentraciones son mayores, así como en el oeste en la salida a Quiroga (Fig. 5).

El Mn supera con mucho los LMP de $1800 \mathrm{mg} / \mathrm{kg}$ de las normas norteamericanas (USEPA 2004). Lu et al. (2007) reportan en Guangzhou, China valores de $479 \mathrm{mg} / \mathrm{kg}, 10$ veces menores a los encontrados en este estudio.

El Li presenta las concentraciones mayores (15 a $18 \mathrm{mg} / \mathrm{kg}$ ) en la parte este, noreste y sureste (Fig.6) pero no rebasa los LMP $(1600 \mathrm{mg} / \mathrm{kg})$ de la norma USEPA (2004).

\section{Mapas de los umbrales de contaminación de los EPT}

Otra forma de mostrar la distribución espacial de la contaminación de manera más específica es mostrando las zonas con mayor probabilidad (nivel de 0.8 - 1) de que los EPT excedan los LMP de la NOM 147-SEMARNAT/SSA1-2004 (SEMARNAT 2007) y USEPA (2004). 

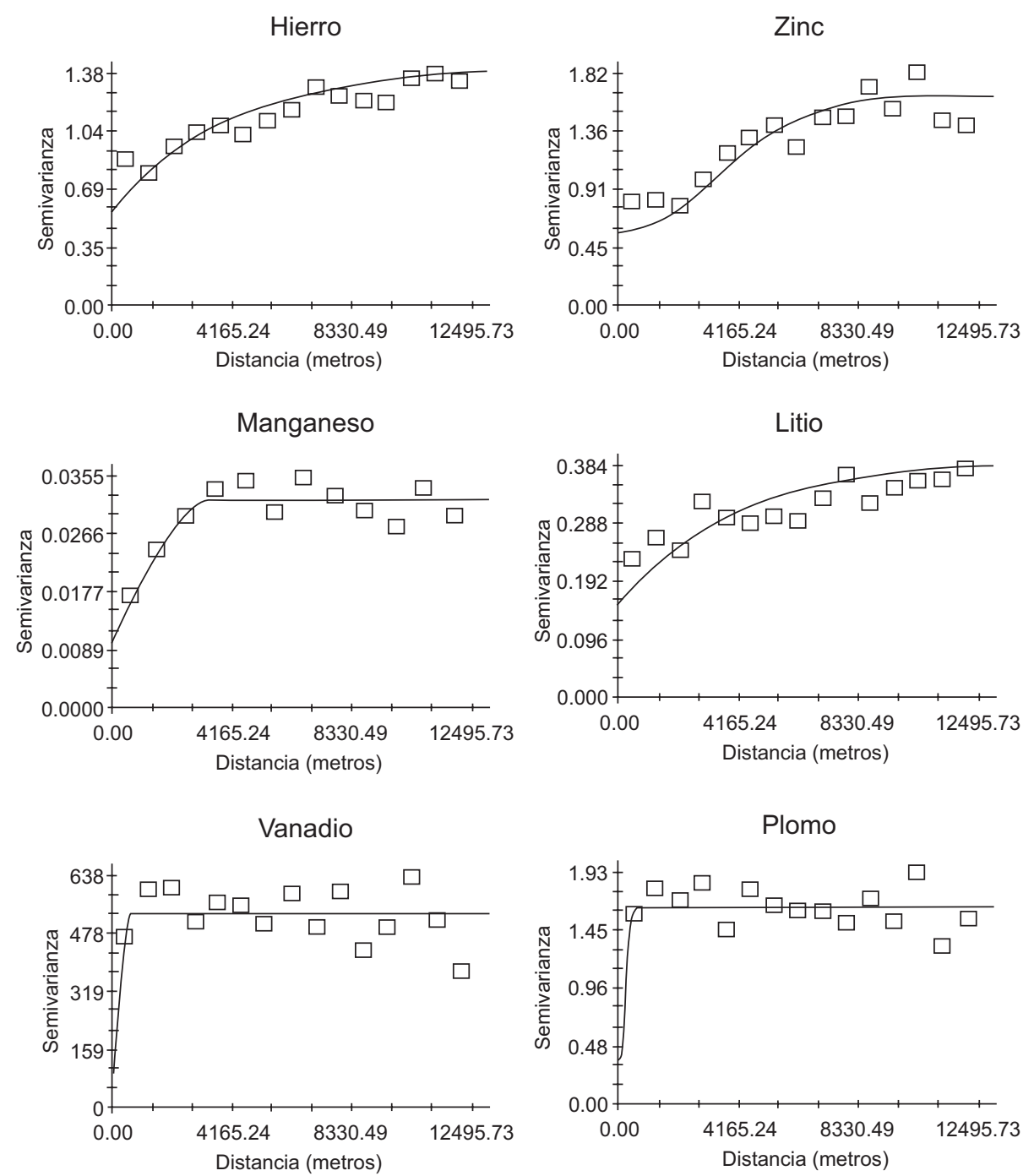

Fig. 2. Modelos de los variogramas de los elementos potencialmente tóxicos en suelos urbanos de Morelia, Michoacán, México. Exponencial en el Li y Fe, esférico en el Mn y V, gaussiano en el $\mathrm{Zn}$ y $\mathrm{Pb}$

El mapa del Fe presenta el nivel más alto $(0.8$ a 1) de probabilidad de rebasar los LMP en el este de la ciudad, en el centro y en una pequeña zona en el oeste; con una superficie total de $14.49 \mathrm{~km}^{2}$ correspondiente al $10 \%$ de la superficie muestreada (Fig. 7,

\section{Cuadro II).}

El mapa del Mn presenta el nivel más alto en el suroeste de la ciudad; abarca una superficie de $1 \mathrm{~km}^{2}$ que corresponde al $0.69 \%$ de la superficie total muestreada (Fig. 8, Cuadro II).

El mapa del $\mathrm{Pb}$ presenta el nivel más alto en el centro de la ciudad, por lo que seguramente su origen es antrópico; abarca una superficie de $0.25 \mathrm{~km}^{2}$ que corresponde al $0.17 \%$ de la superficie muestreada (Fig. 9, Cuadro II).

El mapa del V presenta el nivel más alto en el centro-norte y al este de la ciudad en pequeñas áreas. En estas zonas se encuentran densidades de población mediana y alta con alto flujo vehicular, por lo que seguramente el $\mathrm{V}$ es de origen antrópico. Abarca una superficie de $2.23 \mathrm{~km}^{2}$ que corresponde al $1.54 \%$ de la superficie muestreada (Fig. 10, Cuadro II).

El mapa que integra al $\mathrm{V}, \mathrm{Pb}, \mathrm{Fe}$ y $\mathrm{Mn}$ con los niveles de mayor probabilidad ( 0.8 a 1$)$ de rebasar los LMP se presenta en la figura 11. 

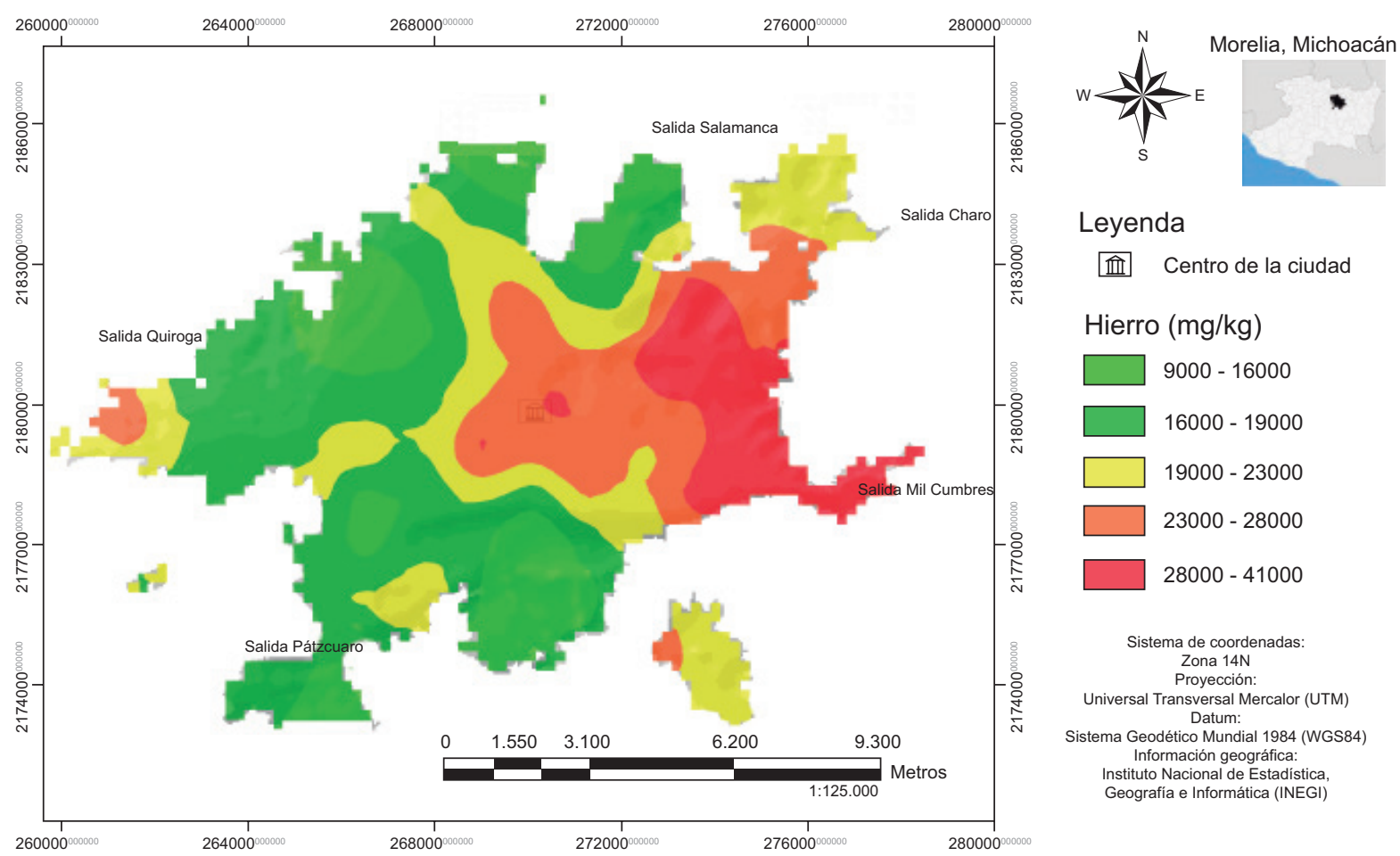

Leyenda

盈 Centro de la ciudad

Hierro $(\mathrm{mg} / \mathrm{kg})$

$9000-16000$

$16000-19000$

$19000-23000$

ए $23000-28000$

$\square 28000-41000$

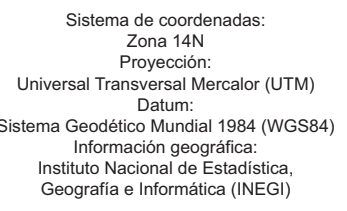

Fig. 3. Distribución espacial del Fe en los suelos urbanos de Morelia, Michoacán, México
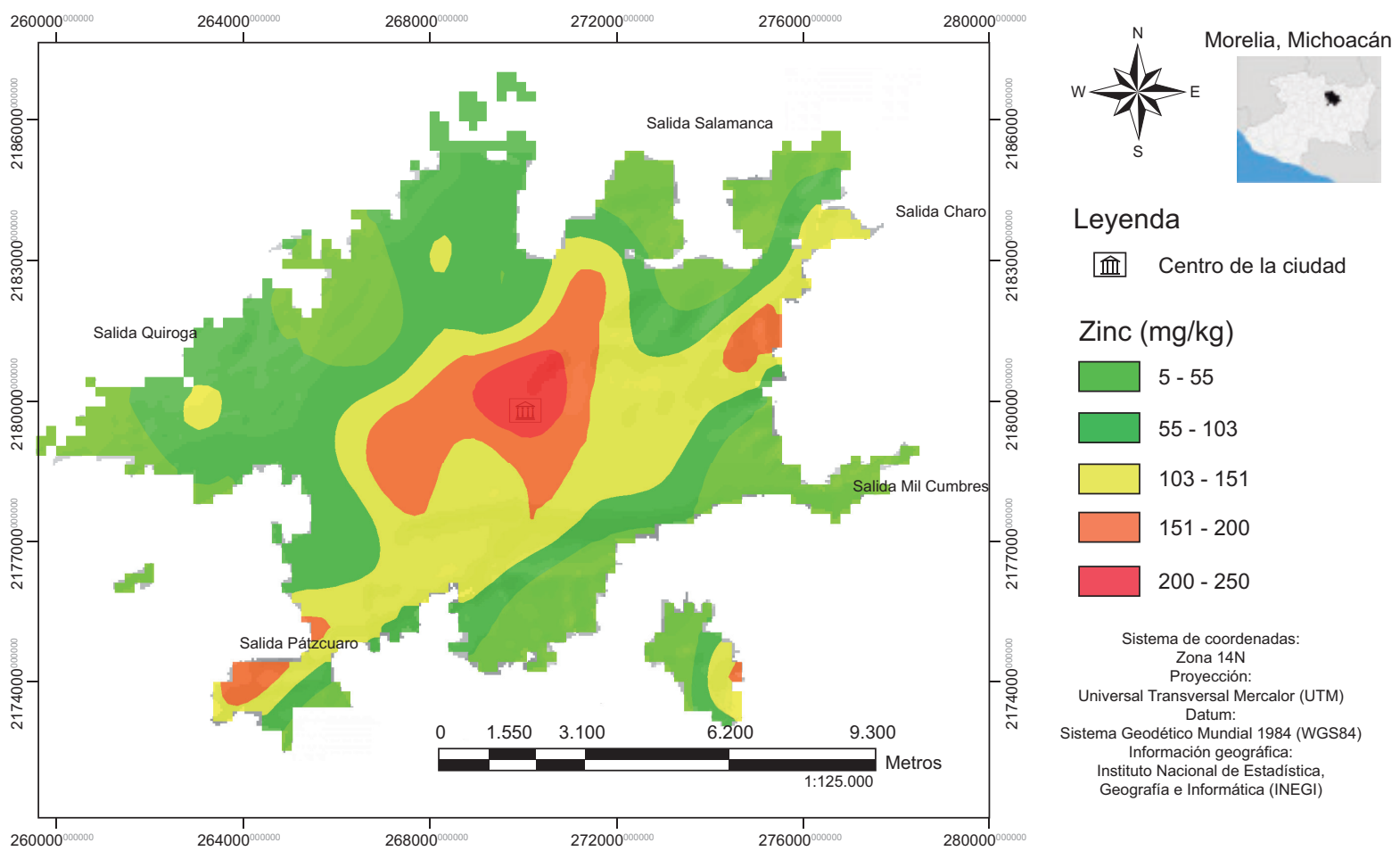

Leyenda

奥 Centro de la ciudad

Zinc $(\mathrm{mg} / \mathrm{kg})$

$\square-55$

$\square 5-103$

$\square 103-151$

$\square 151-200$

$200-250$
Sistema de coordenadas: Zona $14 \mathrm{~N}$
Proyección:
Proyección:
Universal Transversal Mercalor (UTM) Datum:
Sistema Geodético Mundial 1984 (WGS84) Información geográfica:
Instituto Nacional de Estadistica, Geografía e Informática (INEGI)

Fig. 4. Distribución espacial del Zn en los suelos urbanos de Morelia, Michoacán, México 

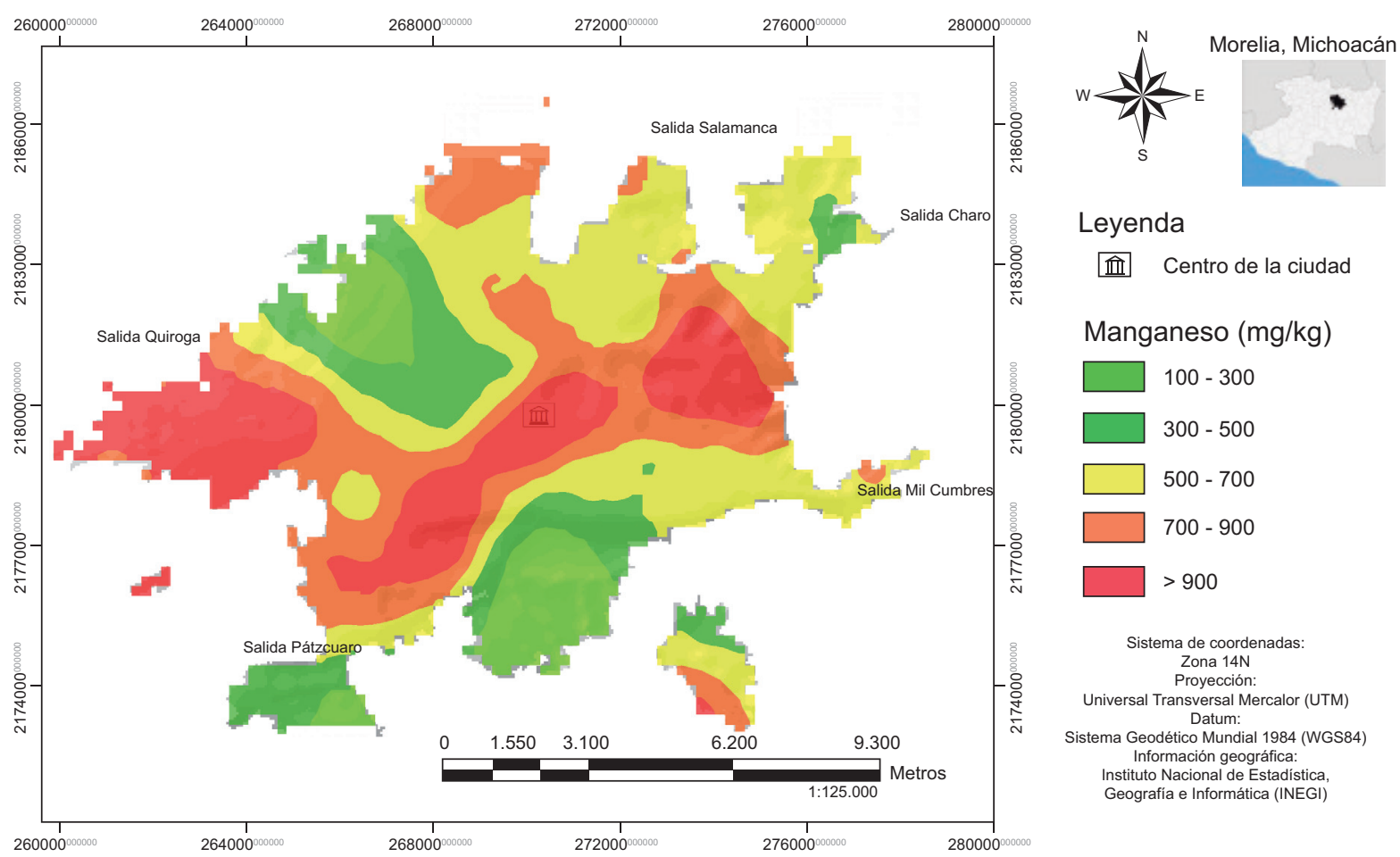

\section{Leyenda}

命 Centro de la ciudad

\section{Manganeso $(\mathrm{mg} / \mathrm{kg})$}

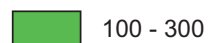

$300-500$

$\square 500-700$

$700-900$

$>900$

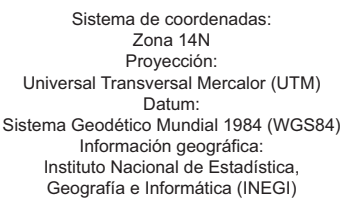

Fig. 5. Distribución espacial del Mn en los suelos urbanos de Morelia, Michoacán, México
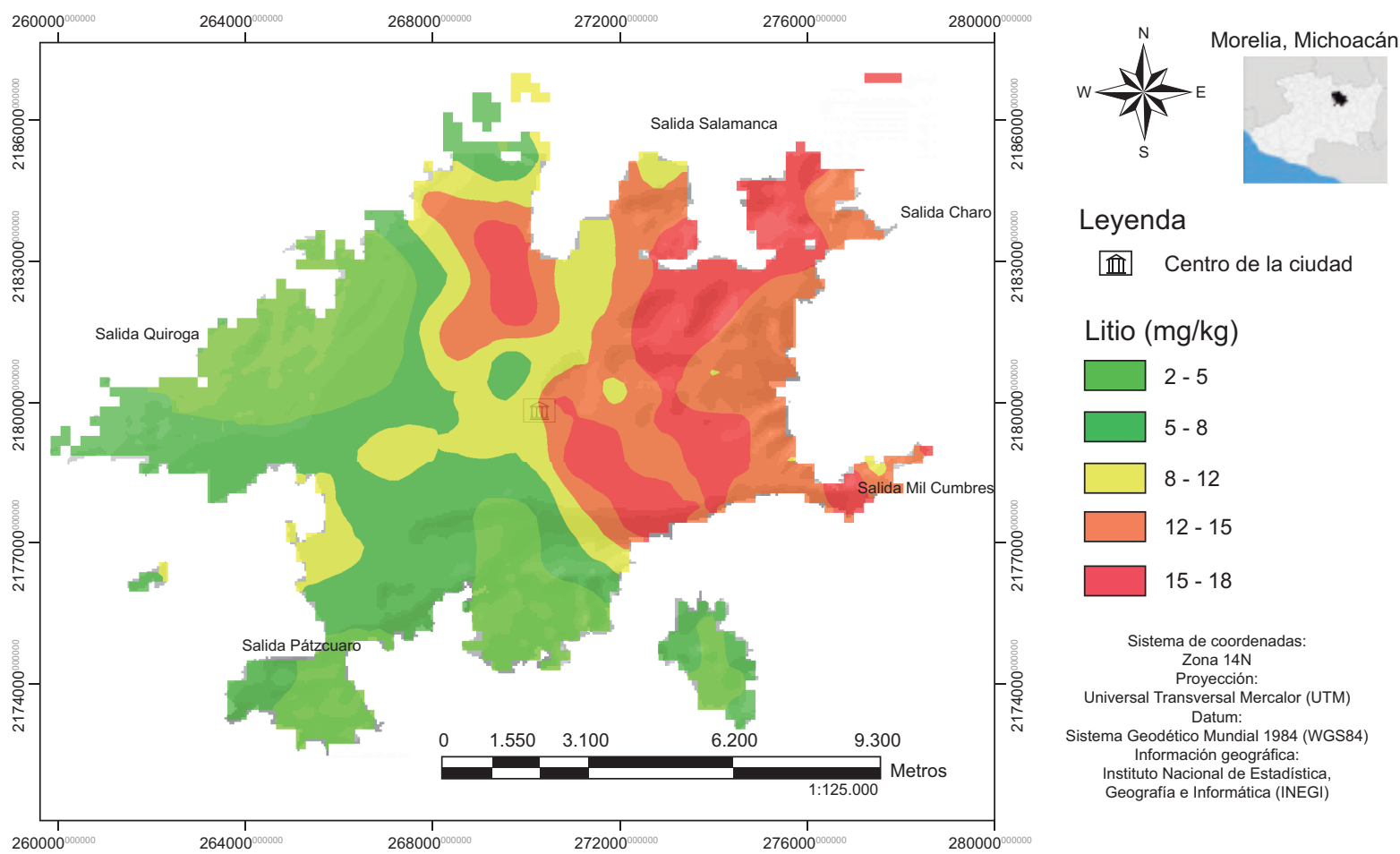

Leyenda

血 Centro de la ciudad

Litio $(\mathrm{mg} / \mathrm{kg})$

$2-5$

ए 5 - 8

$\square-12$

$12-15$

$15-18$

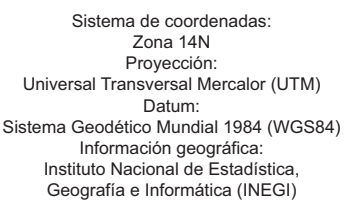

Fig. 6. Distribución espacial del Li en los suelos urbanos de Morelia, Michoacán, México 


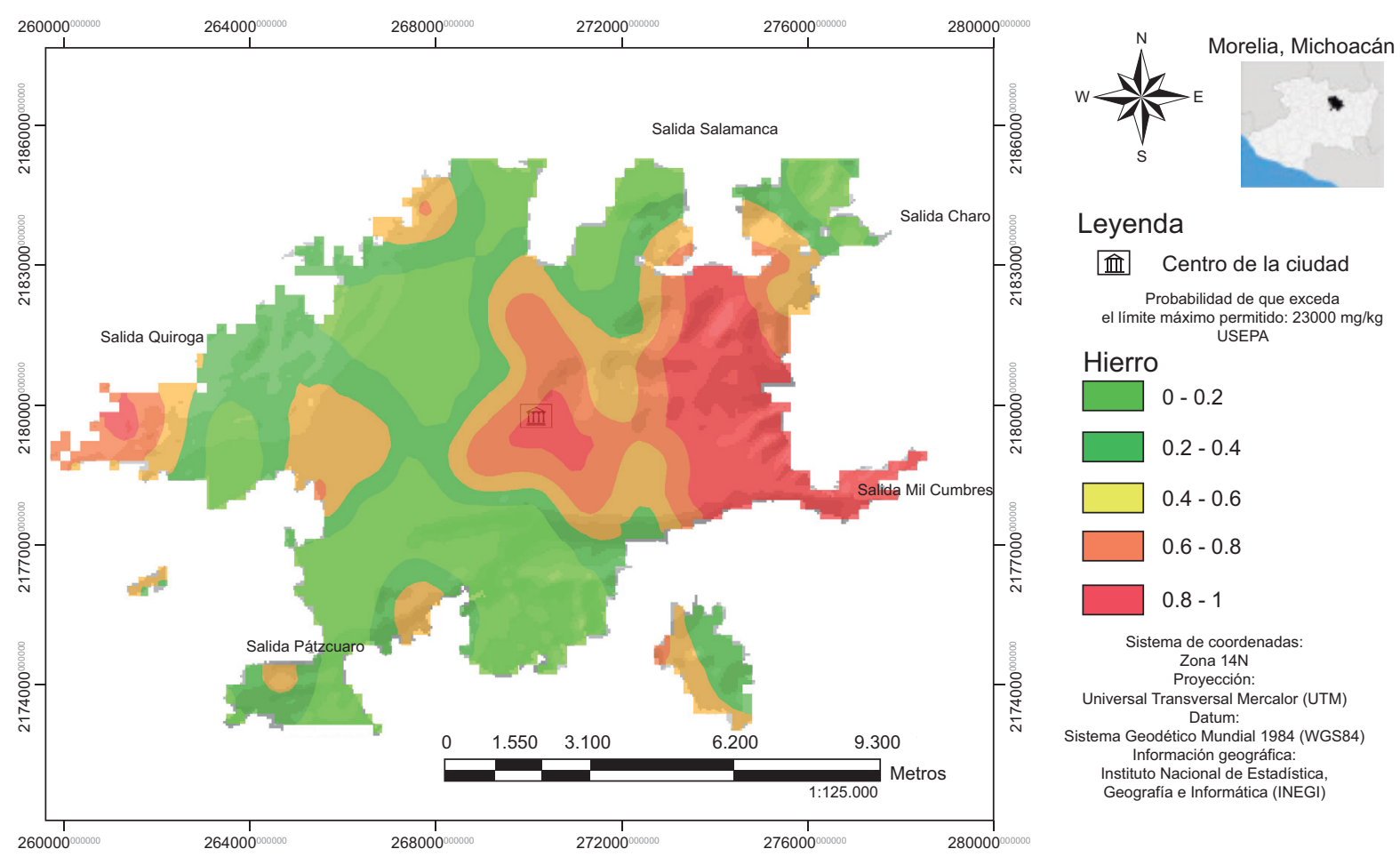

Fig. 7. Distribución del Fe en los suelos urbanos de Morelia, Michoacán, México con los niveles de probabilidad de rebasar los límites máximos permitidos por la norma USEPA (2004)

El mostrar las zonas de intersección de los EPT con las mayores probabilidades de rebasar los LMP, permite señalar las áreas prioritarias para atenderse con acciones de remediación. Se distribuyen de la siguiente manera: $\mathrm{Pb}$ y $\mathrm{Fe}$ en el centro de la ciudad y el Fe y V en el este.

$\mathrm{El}$ área de intersección total del Fe-Pb y el Fe-V es menor de $1 \%$ de la superficie muestreada (Cuadro II). Cortés et al. (2017) reportan en Ensenada Baja California, México, un área de intersección de EPT en polvos urbanos correspondiente al $21.26 \%$.

\section{Perspectivas}

Este estudio permitió identificar las zonas contaminadas que deben ser atendidas por las autoridades y es la primera parte de diversas soluciones a la problemática de contaminación de suelos urbanos de Morelia. Las siguientes propuestas complementarán las soluciones al problema:

a) realización de estudios de las fracciones químicas de los EPT, del tamaño de las partículas del suelo y su contenido de EPT.

b) evaluación de la capacidad amortiguadora de los suelos (Campuzano et al. 2017).
CUADRO II. INTEGRACIÓN DE LA SUPERFICIE CONTAMINADA CON ELEMENTOS POTENCIALMENTE TÓXICOS EN LOS SUELOS URBANOS DE MORELIA, MICHOACÁN, MÉXICO

\begin{tabular}{lcc}
\hline EPT & Superficie $\left(\mathrm{km}^{2}\right)$ & Porcentaje (\%) \\
\hline Vanadio & 2.23 & 1.54 \\
Plomo & 0.25 & 0.17 \\
Hierro & 14.49 & 9.99 \\
Manganeso & 1.00 & 0.69 \\
Área contaminada total & 17.94 & 12.39 \\
Área total & 144.96 & 100 \\
\hline
\end{tabular}

EPT $=$ elementos potencialmente tóxicos

c) desarrollo y evaluación de técnicas de diagnóstico rápido y preciso como el magnetismo edáfico (Cejudo et al. 2015) y el color del suelo (Cortés et al. 2017).

d) identificación del origen antrópico o natural de los EPT mediante diversos factores de contaminación (Wang y Qin 2007, Ihl et al. 2015).

e) el diseño de técnicas de remediación de la contaminación en suelos mediante coloides, modificación del pH y de la estructura edáfica. 

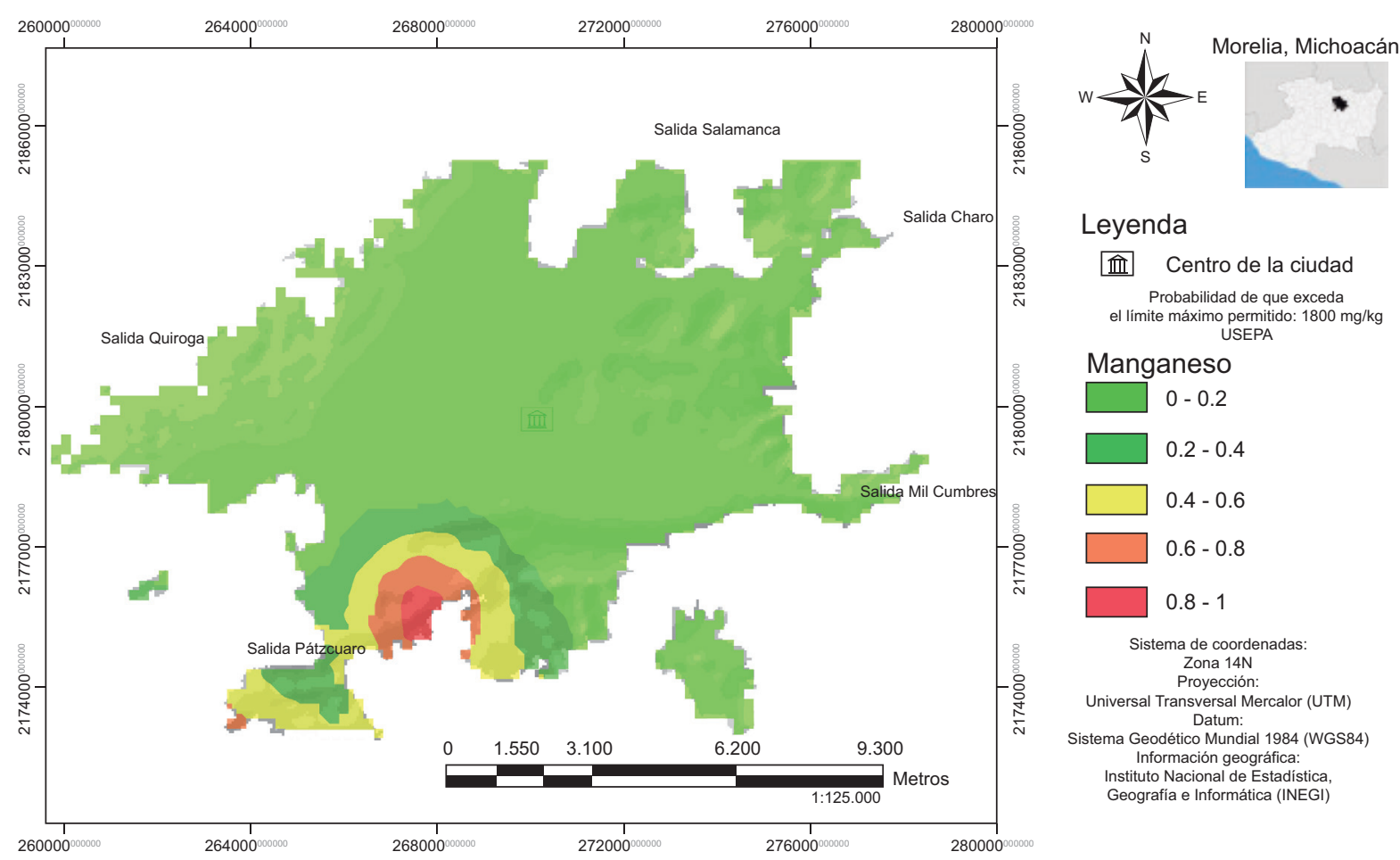

\section{Leyenda}

奥 Centro de la ciudad Probabilidad de que exceda el límite máximo permitido: $1800 \mathrm{mg} / \mathrm{kg}$

\section{Manganeso}

$0-0.2$

$0.2-0.4$

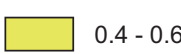

$0.6-0.8$

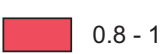

Sistema de coordenadas: Zona $14 \mathrm{~N}$
Proyección

Proyección:
Universal Transversal Mercalor (UTM)

Datum:
Sistema Geodético Mundial 1984 (WGS84) Información geográfica:

Instituto Nacional de Estadística, Geografia e Informática (INEGI)

Fig. 8. Distribución del Mn en suelos urbanos de Morelia, Michoacán, México con los niveles de probabilidad de rebasar los límites máximos permitidos por la norma USEPA (2004)
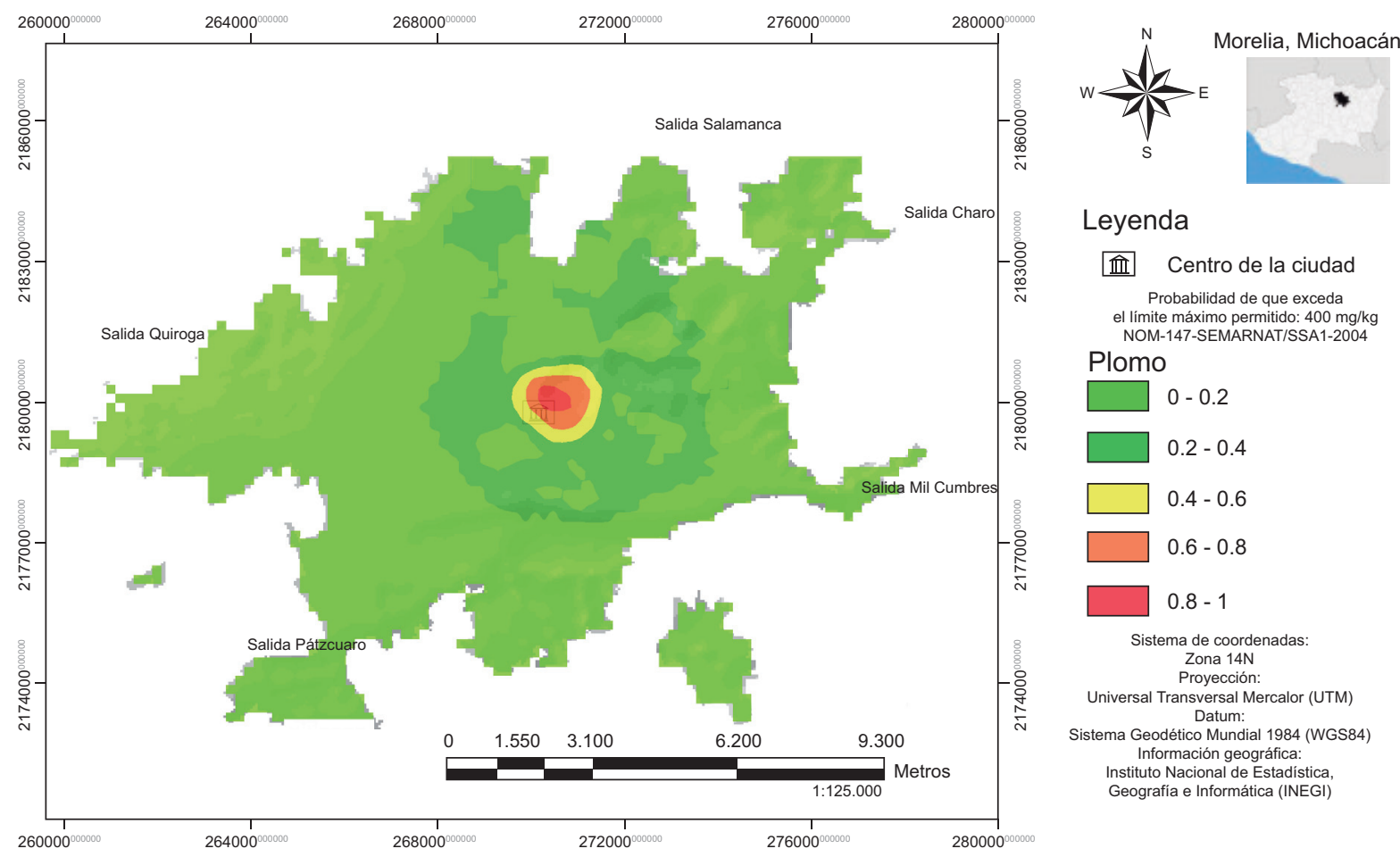

Leyenda

Centro de la ciudad

Probabilidad de que exceda el límite máximo permitido: $400 \mathrm{mg} / \mathrm{kg}$
NOM-147-SEMARNAT/SSA1-2004

Plomo

$0-0.2$

$0.2-0.4$

$\square 0.4-0.6$

$0.6-0.8$

$0.8-1$

Sistema de coordenadas: Zona $14 \mathrm{~N}$

Proyección:
Universal Transversal Mercalor (UTM)

Datum:
Sistema Geodético Mundial 1984 (WGS84) Información geográfica:

Instituto Nacional de Estadística,
Geografía e Informática (INEGI)

Fig. 9. Distribución del $\mathrm{Pb}$ en los suelos urbanos de Morelia, Michoacán, México con los niveles de probabilidad de rebasar los límites máximos permitidos por la NOM-147-SEMARNAT/SSA1-2004 (SEMARNAT 2007) 

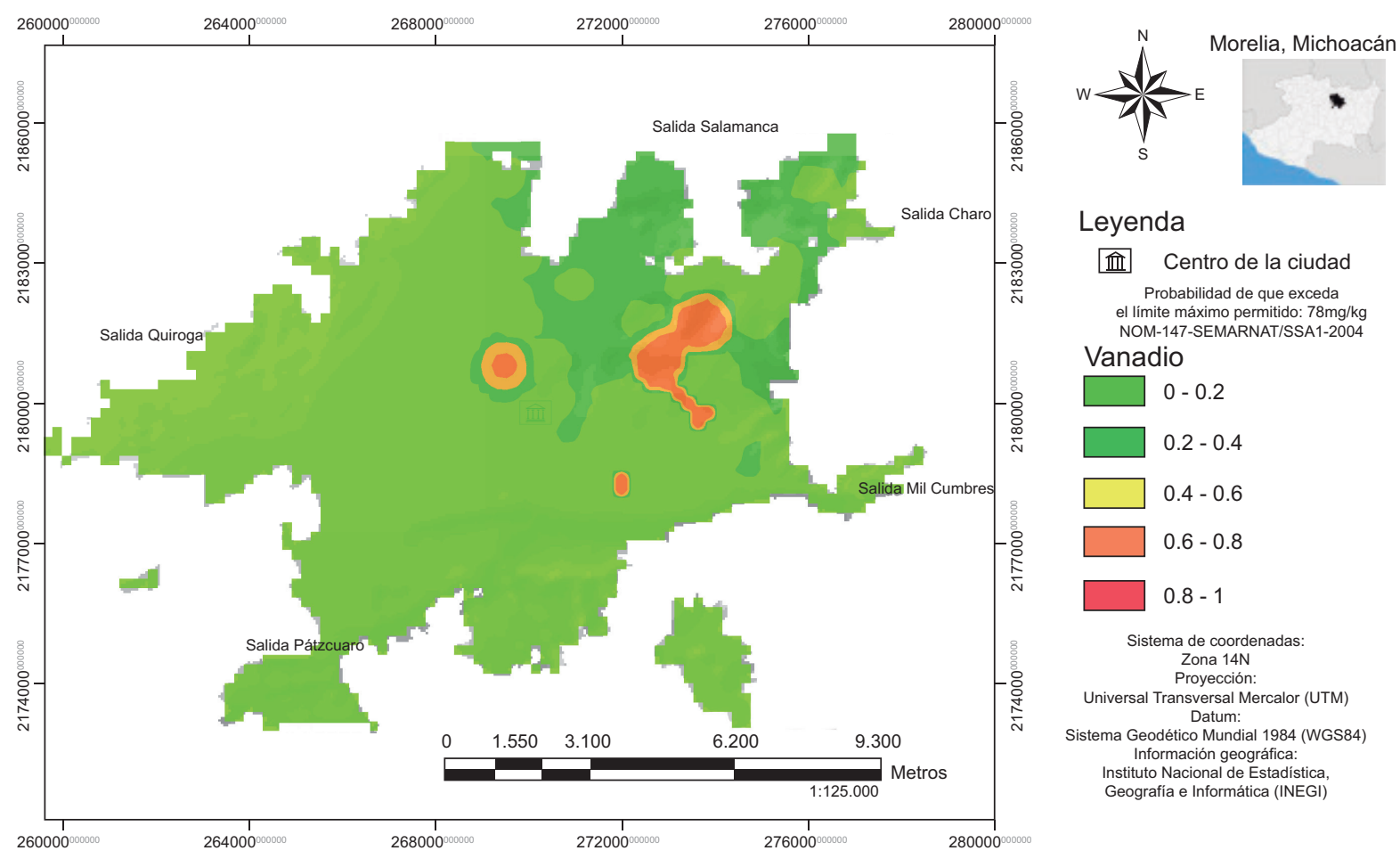

Leyenda

Centro de la ciudad Probabilidad de que exceda el limite máximo permitido: $78 \mathrm{mg} / \mathrm{kg}$ NOM-147-SEMARNAT/SSA1-2004 Vanadio

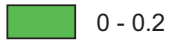

$\square \quad 0.2-0.4$
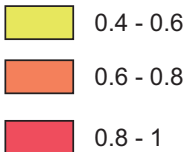

Sistema de coordenadas: Zona $14 \mathrm{~N}$

Proyección:
Universal Transversal Mercalor (UTM) Datum:
Sistema Geodético Mundial 1984 (WGS84) Información geográfica: Instituto Nacional de Estadística, Geografía e Informática (INEGI)

Fig. 10. Distribución del V en los suelos urbanos de Morelia, Michoacán, México con los niveles de probabilidad de rebasar los límites máximos permitidos por la NOM-147-SEMARNAT/SSA1-2004 (SEMARNAT 2007)
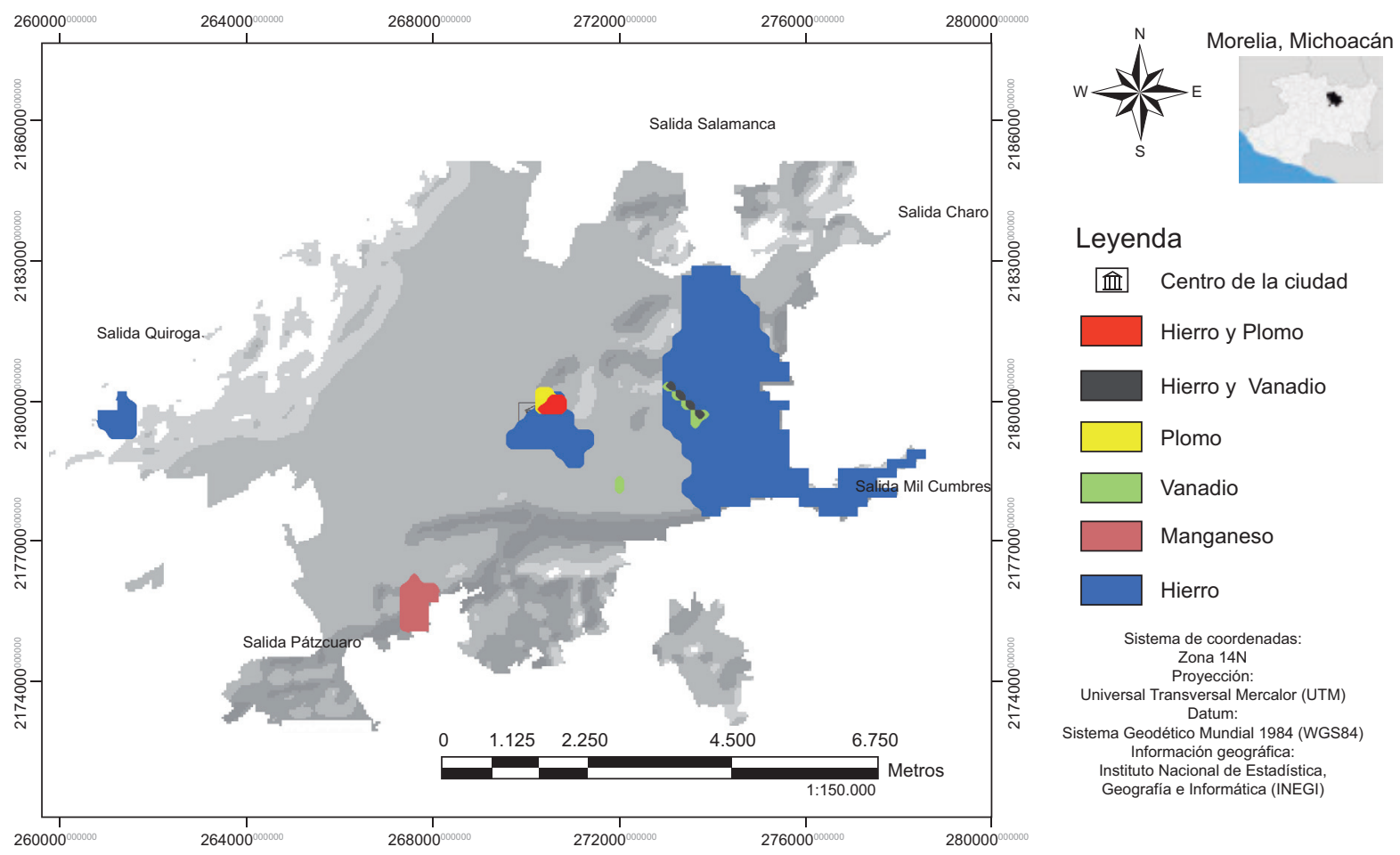

\section{Leyenda}

骨 Centro de la ciudad

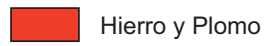

Hierro y Vanadio

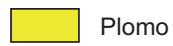

$\square$ Vanadio

Manganeso
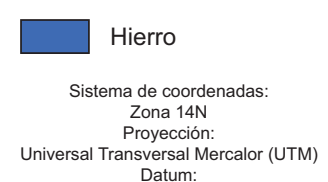

Datum:
Sistema Geodético Mundial 1984 (WGS84) Sistema Geodético Mundial 1984 (WGS84) Información geográfica: Instituto Nacional de Estadística,
Geografia e Informática (INEGI)

Fig. 11. Distribución de la contaminación de suelos con $\mathrm{V}, \mathrm{Pb}, \mathrm{Mn}$ y Fe en los suelos urbanos de Morelia, Michoacán, México con el nivel de probabilidad de 0.8 -1 de rebasar los límites máximos permitidos por la NOM-147-SEMARNAT/SSA1-2004 (SEMARNAT 2007) y la norma USEPA (2004) 


\section{CONCLUSIONES}

La contaminación de los suelos urbanos de la ciudad de Morelia y su zona conurbada está determinada por la presencia del Fe, Mn, Pb, V, Li y Zn. Considerando las concentraciones máximas permisibles los elementos que las rebasan son el Fe, $\mathrm{Mn}, \mathrm{Pb}$ y V, en ese orden de importancia teniendo en cuenta la concentración y el porcentaje de superficie. Las concentraciones del Li y $\mathrm{Zn}$ no rebasaron los valores establecidos por las normas. El este de la ciudad, en donde se ubica la zona industrial más grande, tiene la mayor superficie con suelos contaminados por Fe y en menor proporción por V. El suroeste, en donde se encuentra otra zona industrial, se encuentra contaminada por Mn. El oeste por Fe. El norte es la única zona en donde ninguno de los EPT rebasa las normas. Los suelos del centro de la ciudad están contaminados con $\mathrm{Fe}$ y $\mathrm{Pb}$. Esta zona está rodeada por usos de suelo con la mayor densidad de población por lo que debería tener prioridad para ser atendida.

\section{AGRADECIMIENTOS}

Agradecemos al Consejo Nacional de Ciencia y Tecnología por la beca posdoctoral del primer autor y por el soporte financiero del proyecto CB-201101-169915. A la M en C. Hilda Rivas Solórzano del Centro de Investigación en Geografía Ambiental, Universidad Nacional Autónoma de México, por la asesoría en la digestión de las muestras. Francisco Bautista agradece el apoyo económico de la Dirección General de Asuntos del Personal Académico de la Universidad Nacional Autónoma de México para su estancia sabática en el Centro de Edafología y Biología Aplicada del Segura del Consejo Superior de Investigación Científica, España.

\section{REFERENCIAS}

Aguilar Reyes B., Cejudo R., Martínez-Cruz J., Bautista F., Gogichaishvili A., Carvallo C. y Morales J. (2012). Ficus benjamina leaves as indicator of atmospheric pollution: a reconaissance study. Stud. Geophys. Geod. 56 (3), 879-887. DOI: 10.1007/s11200-011-0265-1

Aguilar Reyes B., Bautista F., Gogichaishvili A., Quintana P., Carvallo C. y Battu J. (2013). Rock-Magnetic properties of topsoils and urban dust from Morelia (>800,000 inhabitants), Mexico: implications for anthropogenic pollution monitoring in Mexico's medium size cities. Geofis. Int. 52 (2), 121-133.
ATSDR (2010). Resúmenes de salud pública Mn, Pb, V. Agencia para sustancias tóxicas y el registro de enfermedades [en línea]. https://www.atsdr.cdc.gov/es/ index.html 02/05/2016.

Campuzano E., Delgado C., Bautista F. y Gogichaishvili A. (2017). Índices de adsorción de metales pesados en suelos urbanos: el caso de Morelia, Michoacán. Bol. Soc. Geol. Mex. 69 (2), 433-445.

Cejudo R., Delgado C., Israde I., Bautista F., Gogichaisvili A. y Morales J. (2015). Identificación de zonas presumiblemente contaminadas por elementos tóxicos por técnicas no convencionales en la ciudad de Morelia, Michoacán. Latinmag Letters 6, 1-6.

Cortés J.L., Bautista F., Delgado C., Quintana P., Aguilar D., García A., Figueroa C. y Gogichaishvili A. (2017). Spatial distribution of heavy metals in urban dust from Ensenada, Baja California, México. Revista Chapingo Serie Ciencias Forestales y del Ambiente 23 (1), 47-60. DOI: 10.5154/r.rchscfa.2016.02.005

Chen T., Zheng Y., Lei M., Huang Z., Wu H., Chen H., Fan K., Yu K., Wu X. y Tian Q. (2005). Assessment of heavy metal pollution in surface soils of urban parks in Beijing, China. Chemosphere 60, 542-551.

DOI: 10.1016/j.chemosphere.2004.12.072

Chen P., Chakraborty S., Mukhopadhyay S., Lee E., Paoliello M.M.B., Bowman A.B. y Aschner M. (2015). Manganese homeostasis in the nervous system. J. Neurochem. 134, 601-610. DOI: 10.1111/jnc.13170

Delgado C., Pacheco A.J., Cabrera S.A., Batllori S.E., Orellana R. y Bautista F. (2010). Quality of groundwater for irrigation in tropical karst environment: The case of Yucatan, Mexico. Agric. Water Manage. 97, 1423-1433. DOI: 10.1016/j.agwat.2010.04.006

Delgado C., Israde I., Bautista F., Gogichaishvili A., Márquez C., Cejudo R., Morales J. y González I. (2015). Metales pesados en suelos urbanos de Morelia, Michoacán: influencia del uso del suelo y del tipo de vialidad. Ciencia Nicolaita 65, 120-138.

ESRI (2004). ArcGIS 9. Getting started with ArcGIS. Environmental Systems Research Institute Inc. ESRI, Redlands, CA, EUA.

Garduño-Monroy V.H., Rodríguez-Torres G.M., IsradeAlcántara I., Arreygue-Rocha E., Canuti P. y Chiesa S. (1999). Efectos del clima El Niño en los fenómenos de fluencia de las fallas geológicas de la ciudad de Morelia. Geos 9 (2), 84-93.

Goovaerts P. (1999). Geostatistics in soil science: Stateofthe-art and perspectives. Geoderma 89, 1-45. DOI: 10.1016/S0016-7061(98)00078-0

Granero S. y Domingo J.L. (2002). Levels of metals in soils of Alcalá de Henares, Spain: Human health risks. Environ. Int. 28, 159-164.

DOI: $10.1016 / \mathrm{S} 0160-4120(02) 00024-7$ 
Hernández-Stefanoni, J.L. y Ponce-Hernández R. (2006). Mapping the spatial variability of plant diversity in a tropical forest: comparison of spatial interpolation methods. Environ. Monit. Assess. 117, 307-334. DOI: 10.1007/s10661-006-0885-z

Ihl T., Bautista F., Cejudo R., Delgado C., Quintana P., Aguilar D. y Gogichaishvili A. (2015). Concentration of toxic elements in topsoils of the metropolitan area of Mexico City: a spatial analysis using Ordinary kriging and Indicator kriging. Rev. Int. Contam. Ambie. 31 (1), 47-62.

INECC-SEMARNAT (2010). Estudio de emisiones y actividad vehicular. Instituto Nacional de Ecología y Cambio Climático-Secretaría de Medio Ambiente y Recursos Naturales. Dirección de Investigación sobre la Contaminación Urbana y Regional (DGICUR), Dirección de Investigación sobre la Calidad del Aire (DICA). Ciudad de México, México, 78 pp.

INEGI (2010). Censos y conteos de población y vivienda, México. Instituto Nacional de Estadística, Geografía e Informática [en línea]. http://www.inegi.org.mx/ lib/olap/consulta/general_ver4/MDXQueryDatos. asp?\#Regreso\&c=27770 29/07/2016.

INEGI (2014). Automóviles registrados en circulación en la ciudad de Morelia Michoacán, México. Instituto Nacional de Estadística, Geografía e Informática [en línea]. http://www3.inegi.org.mx/sistemas/biinegi/ 29/07/2016.

Isaaks E.H. y Srivastava R.M. (1989). An introduction to applied geoestadistics. Oxford University Press. New York, EUA. 561 pp.

Jordao C.P., Nascentes C.C., Cecon P.R., Fontes R.L.F. y Pereira J.L. (2006). Heavy metal availability in soil amended with composted urban solid wastes. Environ. Monit. Assess. 112, 309-326.

DOI: 10.1007/s10661-006-1072-y

Laing J.R., Hopke P.K., Hopke E.F., Husain L., Dutkiewicz V.A., Paatero J. y Viisanen Y. (2014). Long-term particle measurements in Finnish Arctic: Part I. Chemical composition and trace metal solubility. Atmos. Environ. 88, 275-284.

DOI: 10.1016/j.atmosenv.2014.01.015

Lenntech BV (2016). Water treatment solutions. Tabla periódica. Elementos [en línea]. http://www.lenntech. es/periodica/elementos/fe.htm 14/01/2017.

Lu Y., Zhu F., Chen J., Gan H. y Guo Y. (2007). Chemical fractionation of heavy metals in urban soils of Guangzhou, China. Environ. Monit. Assess. 174, 429-439. DOI: $10.1007 / \mathrm{s} 10661-007-9634-1$

Madrid F., Biasioli M. y Ajmone-Marsan F. (2008). Availability and bioaccessibility of metals in fine particles of some urban soils. Arch. Environ. Contam. Toxicol. 55, 21-32. DOI: 10.1007/s00244-007-9086-1
Morton O., Puchelt H., Hernández E. y Lounejeva E. (2001). Traffic-related platinum group elements (PGE) in soils from Mexico City. J. Geochem. Explor. 72, 223-227.

PDUCPM (2010). Cuatro documentos parciales por zonas del estado de Michoacán, México. Programas de Desarrollo Urbano del Centro de Población Morelia [en línea]. www.morelia.gob.mx/pdfs/IMDUM/PPDUZOM 24/10/2012.

Rawlins B.G., Lark R.M., O'Donnell K.E., Tye A.M. y Lister T.R. (2005). The assessment of point and diffuse metal pollution of soils from an urban geochemical survey of Sheffield. Soil Use Manage 21, 353-362. DOI: 10.1079/SUM2005335

Robertson G. P. (2008). GS+: Geostatistics for the environmental sciences. Gamma Design Software. Plainwell, Michigan, EUA, 172 pp.

Sabath E. y Robles-Osorio M.L. (2012). Medio ambiente y riñón: nefrotoxicidad por metales pesados. Nefrología 32 (3), 279-286. DOI: 10.3265/Nefrologia.pre2012. Jan. 10928

Sánchez-Duque A., Bautista F., Gogichaishvili A., Cejudo-Ruiz R., Reyes-López J.A., Solís-Domínguez F.A. y Morales-Contreras J.J. (2015). Evaluación de la contaminación ambiental a partir del aumento magnético en polvos urbanos. Caso de estudio en la ciudad de Mexicali, México. Rev. Mex. Cien. Geol. 32 (3), 501-513.

SEMARNAT (2007). Norma Oficial Mexicana NOM147-SEMARNAT/SSA1-2004. Secretaría de Medio Ambiente y Recursos Naturales. Diario Oficial de la Federación. 2 de marzo de 2007.

Sommer I., Fernández P., Rivas H. y Gutiérrez M. (2000). La geoestadística como herramienta en estudios de contaminación de suelos. Análisis de caso: afectación por arsénico, plomo y cadmio contenidos en jales mineros. Rev. Int. Contam. Ambie. 16 (4), 205-214.

Su D.C. y Wong J.W.C. (2003). Chemical speciation and phytoavailability of $\mathrm{Zn}, \mathrm{Cu}, \mathrm{Ni}$ and $\mathrm{Cd}$ in soil amended with fly ash-stabilized sewage sludge. Environ. Int. 29, 895-900. DOI: 10.1016/S0160-4120(03)00052-7

Testiati E., Parinet J., Massiani C., Laffont-Schwob I., Rabier J., Pfeifer H.R., Lenoble V., Masotti V. y Prudent P. (2013). Trace metal and metalloid contamination levels in soils and in two native plant species of a former industrial site: Evaluation of the phytostabilization potential. J. Hazard. Mater. 248-249, 131-141.

DOI: $10.1016 /$ j.jhazmat.2012.12.039

USEPA (2004). A preliminary remediation goals table. Region 9: The pacific southwest. United States Environmental Protection Agency. (en línea) http://www. epa.gov/region09/waste/sfund/prg/files/04prgtable. pdf 22/08/2015. 
Wang X.S. y Qin Y. (2007). Some characteristics of the distribution of heavy metals in urban topsoil of Xuzhou, China. Environ. Geochem. Health. 29, 11-19. DOI: $10.1007 / \mathrm{s} 10653-006-9052-2$
Webster R. y Oliver M.A. (1990). Statistical methods in soil and land resource survey. Oxford University Press. Nueva York, EUA, 316 pp. 